\title{
Numerical Simulation of a Complete Helicopter Configuration in Forward Flight Using Fluid-Structure Coupling
}

\author{
Walid Khier \\ Institut für Aerodynamik und Strömungstechnik, \\ Deutsches Zentrum für Luft- und Raumfahrt e.V. (DLR) \\ Lilienthalplatz 7, 38108 Braunschweig, Germany \\ email:walid.khier@dlr.de
}

\begin{abstract}
Summary
This paper highlights the results obtained by coupling the aeromechanic tool HOST with the DLR CFD solver FLOWer within the framework of the EUProject GOAHEAD. The process was applied to predict the flow around the GOAHEAD configuration in forward flight at Mach number of 0.204 and fuselage pitch angle of $-2 \cdot 5^{\circ}$. Comparisons of the CFD results with experimental measurements revealed very good agreement for main rotor pressure and the front part of the fuselage. Main rotor power could be predicted with an accuracy of $0.25 \%$.
\end{abstract}

\section{Introduction}

The flowfields around helicopters are characterized by elaborate physics involving strong three-dimensional effects, high degree of unsteadiness, large separated flow areas and complex wake structure. These features make experimental investigation and analysis of helicopter aerodynamics an extremely difficult task. Consequently, the popularity of CFD has evolved rapidly in helicopter research and development over the past few decades. At the present time, CFD analysis based on the solution of Reynolds-Averaged Navier-Stokes (RANS) equations is being applied in industry to study isolated helicopter components like e.g. the main rotor, rotor hub, fenestron, fuselage...etc. 
Although the capabilities of present day CFD tools to predict the flow past a complete helicopter configuration has been demonstrated [1], the lack of suitable experimental database prevented extensive validation of the numerical tools for helicopter aerodynamic application. Therefore, the EU project GOAHEAD was initiated to generate a comprehensive experimental database especially conceived for this specific purpose. The project included a code validation activity consisting of a preliminary (blind) phase and a final (post) phase in parallel to the measurements. An overview of the project and the blind test activities is given by [2] and [3], respectively.

Since rigid blade assumption usually leads to non trivial deviation in the onset of the flow along the blade, a proper simulation of blade dynamics and elastic deformation is essential for an accurate prediction of rotor performance ([4] and [5]). The measured displacement of the blade can be imposed in CFD simulation as in [6], or, instead, the deformation can be computed by a rotor simulation code coupled (loosely or directly) to the CFD solver ([3], [7] and [8]).

In this paper time accurate RANS simulation of the flow past a complete helicopter configuration in medium speed forward flight is reported. Weak fluidstructure coupling was applied to generate predefined thrust and propulsive force, and to obtain the deformation of the blades. The accuracy of the simulation is assessed by comparison with measured data.

\section{Approach}

\section{Numerical Tools}

The DLR finite volume, block-structured solver FLOWer [9] was applied to predict the flow by solving the RANS equations in three dimensions accurately in time using dual time stepping. Turbulence effects were accounted for via a modified version [10] of Wilcox $k-\omega$ mode. FLOWer relies on the Chimera technique to realize the relative motion between the moving and stationary components of the aircraft. The rotor was trimmed to encounter pre-defined weight, lateral and propulsive forces using the stand alone flight mechanics tool HOST [11]. The resulting rotor controls and elastic deformation of the blade surface for the whole radial and azimuth range were then imposed on the CFD simulation to modify the blade surface geometry following the approach presented in ([8] and [12]).

\section{Computational Model and Numerical Grid}

The computational model refers to the tested GOAHEAD model, featuring a $4.1 \mathrm{~m}$ NH90 fuselage model, ONERA 7AD main rotor, reduced scale BO105 tail rotor, a rotor hub, a strut and slip ring fairing inside the $8 \mathrm{~m} \times 6 \mathrm{~m}$ test section 
of the DNW low-speed wind tunnel (Figure 1). The test section was included in the simulation to allow direct comparisons with uncorrected wind tunnel data to be made. Medium speed cruise conditions were simulated at Mach number equal to 0.204 and fuselage pitch angle of $-2.5^{\circ}$.
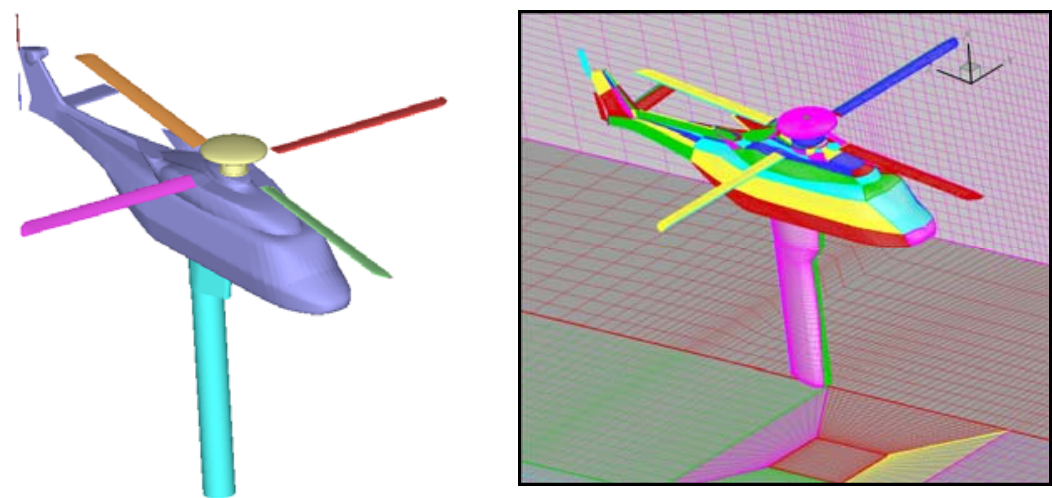

Figure 1: Left: The numerical configuration. Right: Overview of the computational model showing its main components. Wind tunnel section not shown.

Multi-block grids around the different elements were combined via Chimera to build the computational grid with a total number of 25.6 Million points. To eliminate geometrical discrepancies observed between blind test data and experimental measurements, the numerical grid was generated based on 3D scan of the wind tunnel model. Figure 1 depicts the surface grid for the complete helicopter configuration. No-slip wall boundary conditions were applied on all solid surfaces of the helicopter, and slip wall conditions on tunnel walls. Flow variables at inflow and outflow boundary were derived from one-dimensional characteristic theory.

\section{Results}

The fuselage is equipped with a total number of 130 unsteady pressure sensors. A global impression of the quality of prediction can be deduced from the comparison between computed surface pressure and the experiment at azimuth position $\Psi=0^{\circ}$ shown in Figure 2. It should be noted that the main rotor rotates in clockwise direction as seen from above. The upper vertical tail rotor blade is advancing and the lower vertical blade is retreating.

The original experimental data is characterized by strong oscillations of high frequency. The experimental data shown in the figure were obtained by averaging the pressure signals recorded for each azimuthal position over 130 revolutions. 
Qualitatively, good agreement could be obtained over the entire surface. The numerical results clearly reproduce the experimental trend showing a pressure rise on the front end followed by suction peaks on the top and sides of the windshield corresponding to local flow acceleration. The pressure levels on the tail gate are faithfully reproduced.
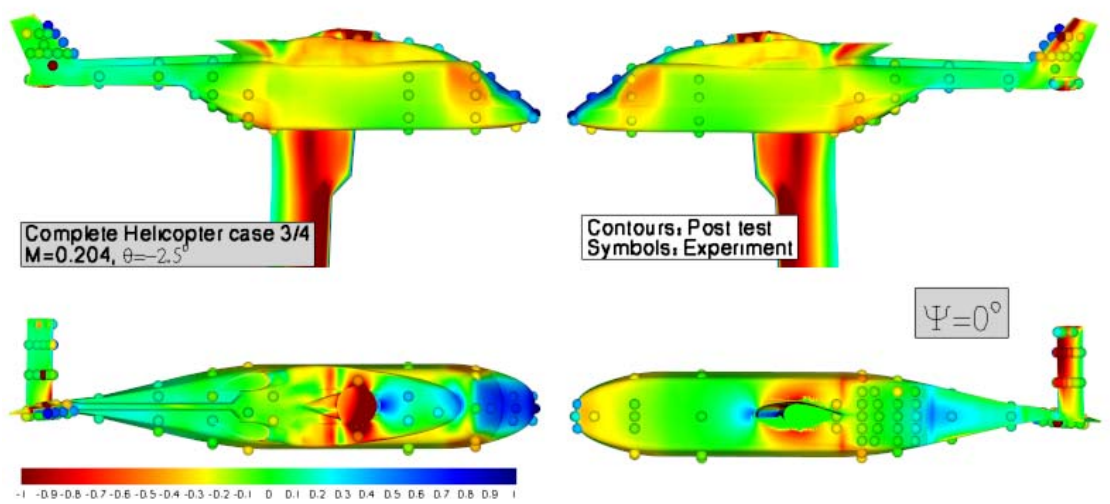

Figure 2: Computed surface pressure (contours) compared with measured pressure (spherical symbols). $\mathrm{M}=0.204, \alpha_{\text {fuselage }}=-2.5^{\circ}$ at main rotor azimuth $\Psi=0^{\circ}$.

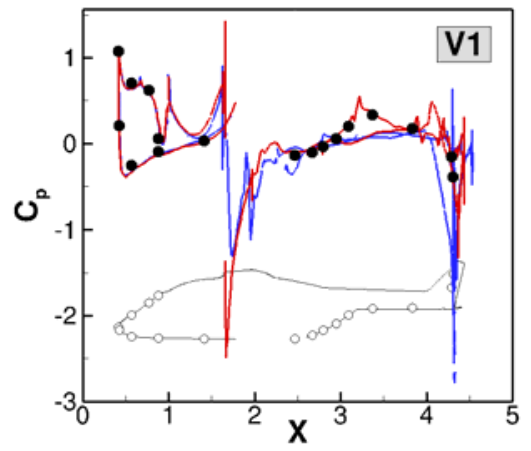

Figure 3: Comparison between computed and measured surface pressure at symmetry. Red curves: post-test results. Blue curves: blind test (not reported here). Black symbols: measurements. $M=0.204, \alpha_{\text {fuselage }}=-2.5^{\circ}$ at main rotor azimuth $\Psi=0^{\circ}$.

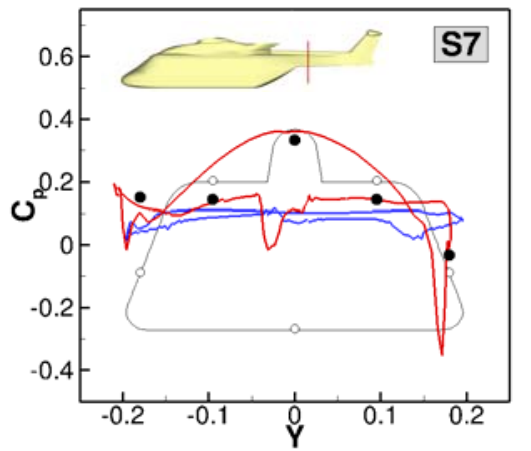

Figure 4: Comparison between computed and measured surface pressure at section S7. Red curves: post-test results. Blue curves: blind test (not reported here). Black symbols: measurements. $\mathrm{M}=0.204$, $\alpha_{\text {fuselage }}=-2.5^{\circ}$ at main rotor azimuth $\Psi=0^{\circ}$.

Figure 3 and Figure 4 show pressure distributions at symmetry plane and cross section S7 of the tail boom, respectively, for the same azimuth position $\left(\Psi=0^{\circ}\right)$. The blue curves in the figure denote results of the blind test phase, 
which are not reported here, and included as a reference only. Figure 3 shows evident improvement in the agreement with the experiment between blind and post test results in the dog house area. Initially a bluff strut fairing was intended for the measurements and has been included in the blind test computations. This led to the observed discrepancy in the dog house. Comparison between blind and post test results shown in Figure 4 confirms and emphasizes this observation. From the figure it can be clearly seen that the post test computations predict the pressure rise on the lower side of the tail boom as well as the pressure asymmetry observed in the experiment. The observed improvements are mainly attributed to using the correct strut in the post test computations, and partially due to grid improvement, especially at the corners.

Broadly good agreement between the computations and measurements can be observed for the sensors on the nose (Figure 5) and on the windscreen (Figure 6). The influence of the rotor is well captured in the computations in terms of frequency and phase, where a clear $4 / \operatorname{Rev}$ signal can be easily seen. As for the magnitude, the agreement varies from good to very good in Figure 5. In Figure 6 the post test results are very hard to identify as they lie too close to the experimental data, indicating an outstanding agreement for the advancing side sensor.

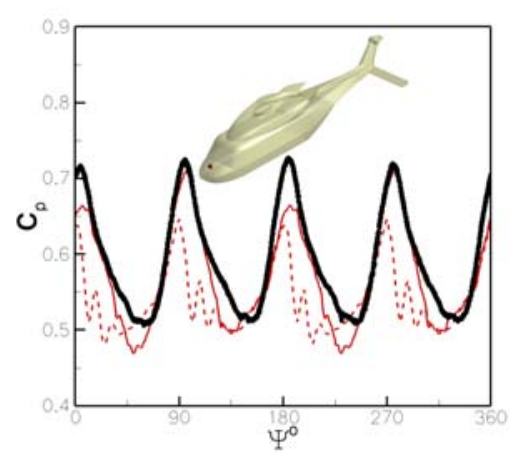

Figure 5: Computed and measured surface pressure signals. Red curve: post-test results. Dashed curve: blind test (not reported here). Black symbols (thick line): measurements. $\mathrm{M}=0.204, \alpha_{\text {fuselage }}=-2.5^{\circ}$

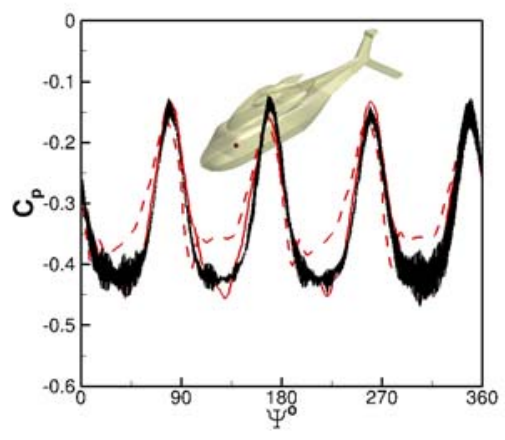

Figure 6: Computed and measured surface pressure signals. Red curve: post-test results. Dashed curve: blind test (not reported here). Black symbols (thick line): measurements. $\mathrm{M}=0.204, \alpha_{\text {fuselage }}=-2.5$

Snap shots of sectional pressure distribution for the main rotor at selected radial and azimuthal positions are illustrated in Figure 7. Solid and dashed curves respectively refer to post and blind test results, while symbols refer to measured data. Qualitatively, the computations are very well in line with the experiment. At $\Psi=30^{\circ}$ there is a slight improvement in the post test results. The difference between blind and post test data at $\Psi=210^{\circ}$ is of the same order as the discrep- 
ancy in the measurements between the different blades. Thus, it is not easy to judge the differences between blind and post test computations. However, both sets of CFD results match well the experimental data.
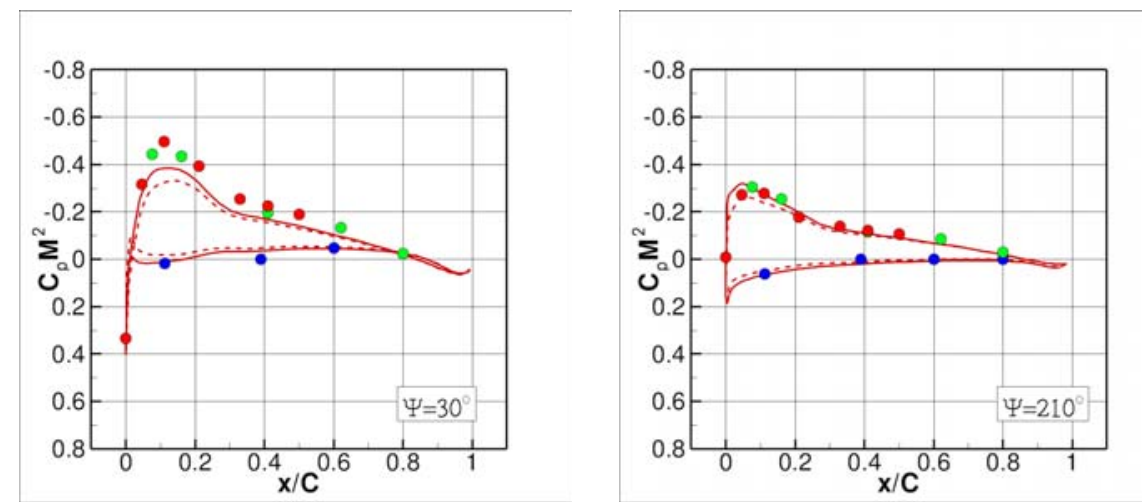

Figure 7: Sectional surface pressure data $\left(C_{p} M^{2}\right)$ for the main rotor at $r / R=0.825 . M=0.204$, $\alpha_{\text {fuselage }}=-2.5^{\circ}, \alpha_{\text {shaft }}=-7.5^{\circ}$. Dashed lines: blind test. Solid lines: final computations. Symbols: experiment (different colors denote different blades). Left: $\Psi=30^{\circ}$, Right: $\Psi=210^{\circ}$

Tail rotor pressures at the radial locations $\mathrm{r} / \mathrm{R}=0.97$ are presented in Figure 8 . Similar to the main rotor data, sectional pressure plots are shown for the azimuthal positions $\Psi=30^{\circ}$ and $210^{\circ}$. In the post test, computations were performed using the experimental pitch values while the flap motion was described as in the blind test matrix. This obviously impaired the accuracy of predictions below the level of the blind test computations reported in [13], and as seen for $\Psi=210^{\circ}$. The improvement observed for the $\Psi=30^{\circ}$ therefore is merely a coincidence, and thus, cannot be explained.
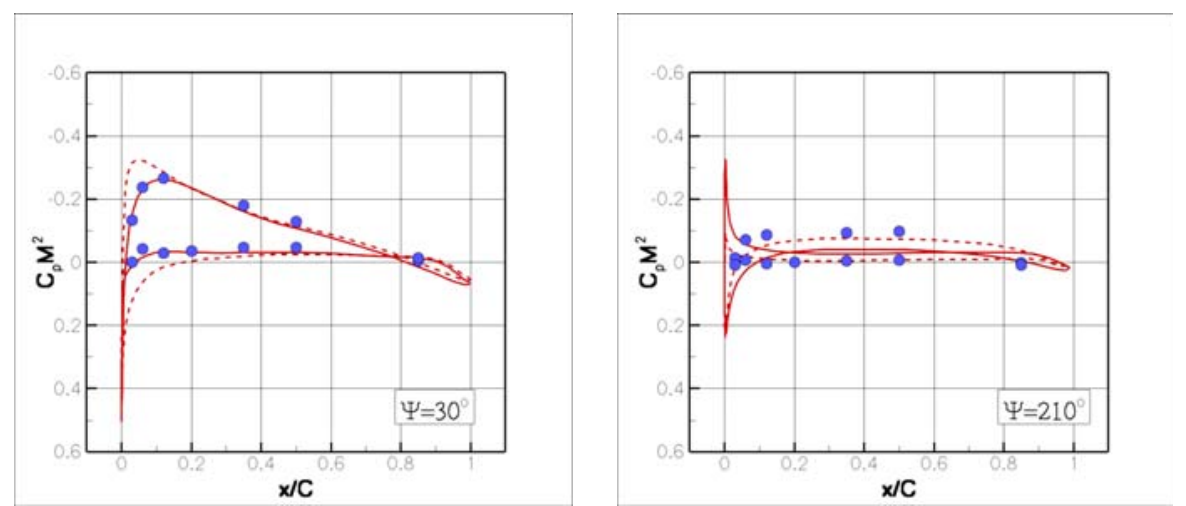

Figure 8: Computed and measured tail rotor sectional pressure at $\mathrm{r} / \mathrm{R}=0.97 . \mathrm{M}=0.204$, $\alpha_{\text {fuselage }}=-2.5^{\circ}, \alpha_{\text {shaft }}=-7.5^{\circ}$. Dashed lines: blind test. Solid lines: final computations. Symbols: experiment. Left: $\Psi=30^{\circ}$, Right: $\Psi=210^{\circ}$ 
Four coupling iterations were required in both the blind and post test calculations for the trim to converge. Converged values of main rotor pitch, flap and lead-lag angles $(\theta, \beta, \delta)$ and their harmonic components are given in

\begin{tabular}{cccccccccc}
\hline \hline & $\theta_{0}\left[{ }^{0}\right]$ & $\theta_{c}\left[^{0}\right]$ & $\left.\theta_{S}{ }^{0}\right]$ & $\beta_{0}\left[{ }^{0}\right]$ & $\beta_{c}\left[^{0}\right]$ & $\beta_{s}\left[^{0}\right]$ & $\delta_{0}\left[^{0}\right]$ & $\delta_{c}\left[{ }^{0}\right]$ & $\delta_{s}\left[^{0}\right]$ \\
\hline Blind & 12.84 & 1.106 & -6.586 & -1.951 & -.202 & 1.141 & -0.306 & 0.205 & -0.86 \\
Final & 13.37 & 1.228 & -6.6 & -1.952 & 0.368 & 1.051 & -0.253 & 0.205 & -0.121 \\
\hline \hline
\end{tabular}

Table 1: Converged blind and final test rotor pitch, flap and lead-lag displacement at blade root

Simplified aerodynamics used in comprehensive codes obviously underestimates power consumption. Fluid-structure coupling considerably improves the power prediction with an error of $0.24 \%$ only, while HOST calculations, even when aeroelastic effects were considered, resulted in $84.9 \%$ of the measured power.

\section{Conclusions}

Results of the blind and post test time accurate RANS simulation of the GOAHEAD configuration under cruise conditions were presented and evaluated. Weak fluid-structure coupling was iteratively applied to trim the main rotor. Good agreement between computed and measured pressure signals on the front upper part of the fuselage in terms of phase and magnitude could be found.

Fluid-structure-flight mechanics coupling proved to be an essential requirement for accurate prediction of the pressure on the rotor. The agreement between computed and measured pressure on the main rotor has improved significantly compared to the results presented in [1].

Accurate representation of the aircraft's geometry is essential for accurate CFD predictions. The strut geometry was found to have a significant influence on the wake structure and the associated surface pressure in the dog house region.

RANS simulation coupled to structure and flight mechanics is an essential approach for accurate prediction of main rotor power requirements. Coupled simulation predicted the power with an accuracy of $0.24 \%$. Aeroelastic rotor simulation with the stand-alone comprehensive code (HOST) underestimated the power consumption by $15.1 \%$. 


\section{References}

[1] Khier, W., Schwarz, T., Raddatz, J., Time-accurate simulation of the flow around the complete Bo105 wind tunnel model. Proceedings of the $31^{\text {st }}$ European Rotorcraft Forum, Florence, Italy, September 2005.

[2] Boelens, O.J., et. al., The blind-test activity of the GOAHEAD project. Proceedings of the $33^{\text {rd }}$ European Rotorcraft Forum, Kazan, Russia, September 2007.

[3] Khier, W., Dietz, M., Schwarz, T., and Wagner, S., Trimmed CFD simulation of a complete helicopter configuration, Proceedings of the $33^{\text {rd }}$ European Rotorcraft Forum, Kazan, Russia, September 2007.

[4] Beaumier, P., Chelli, E., Pahlke, K., Navier-Stokes prediction of helicopter rotor performance in hover including aero-elastic effects, $56^{\text {th }}$ Annual Forum of the American Helicopter Society, Virginia Beach, Virginia, May 2000.

[5] Pahlke, K., Van der Wall, B., Calculation of multi-bladed rotors in high speed forward flight with weak fluid-structure coupling. Proceedings of the $27^{\text {th }}$ European Rotorcraft Forum, Moscow, Russia, September 2001.

[6] Khier, W., Biava, M. and Vigevano, L., CFD Prediction of air flow past a full helicopter configuration. Proceedings of the $36^{\text {th }}$ European Rotorcraft Forum, Paris, France, September 2010.

[7] Dietz, M., Khier, W., Wagner, S. and Krämer, E., Numerical simulation of a full helicopter configuration using weak fluid-structure coupling. $46^{\text {th }}$ AIAA Aerospace Sciences Meeting and Exhibit, Reno, Nevada, $7^{\text {th }}-10^{\text {th }}$ January 2008, AIAA 2008-401.

[8] Dietz, M., Krämer, E., Wagner, S., Altmikus, A.: Weak coupling for active advanced rotors. Proceedings of the $31^{\text {st }}$ European Rotorcraft Forum, Florence, Italy, September 2005.

[9] Kroll, N., Eisfeld, B., Bleecke, H.M., The Navier-Stokes Code FLOWer. Volume 71 of Notes on Numerical Fluid Mechanics, pp. 58-71. Vieweg, Braunschweig, 1999.

[10] Rudnik, R., Untersuchung der Leistungsfähigkeit von ZweigleichungsTurbulenzmodellen bei Profilumströmungen, Deutsches Zentrum für Luft- und Raumfahrt e.V., FB 97-49.

[11] Benoit, B., Dequin, A-M., Kampa, K., Grünhagen, W. v., Basset, P-M., Gimonet, B., HOST: A general helicopter simulation tool for Germany and France. $56^{\text {th }}$ Annual Forum of the American Helicopter Society, Virginia Beach, Virginia, May 2000.

[12] Dietz, M., Keßler, M., Krämer, E., Trimmed simulation of a complete helicopter configuration using fluid-structure coupling, high performance computing in science and engineering 2007, Springer Verlag, 2007.

[13] Khier, W., Numerical Simulation of Air Flow Past a full Helicopter Configuration, Proceedings of the $35^{\text {th }}$ European Rotorcraft Forum, Hamburg, Germany, September 2009. 